\title{
Characterizing the Physical and Thermal Properties of Planetary Regolith at Low Temperatures
}

\author{
James G. Mantovani ${ }^{1}$, Adam Swanger ${ }^{2}$, Ivan I. Townsend III ${ }^{3}$, Laurent Sibille ${ }^{4}$, and \\ Gregory Galloway ${ }^{5}$ \\ ${ }^{1}$ NASA Kennedy Space Center, Surface Systems, Kennedy Space Center, FL 32899; \\ Email: james.g.mantovani@nasa.gov \\ ${ }^{2}$ NASA, Fluids Test \& Tech. Development Branch, Kennedy Space Center, FL 32899 \\ ${ }^{3}$ Craig Technologies (ESC), Kennedy Space Center, FL 32899 \\ ${ }^{4}$ EASI (ESC), Applied Sciences and Technology, Kennedy Space Center, FL 32899 \\ ${ }^{5}$ NASA Kennedy Space Center, Surface Systems, Kennedy Space Center, FL 32899
}

\section{ABSTRACT}

The success or failure of in-situ resource utilization for planetary surface exploration-whether for science, colonization, or commercialization-relies heavily on the design and implementation of systems that can effectively process planetary regolith and exploit its potential benefits. In most cases, this challenge necessarily includes the characterization of regolith properties at low temperatures (cryogenic). None of the nearby solar system destinations of interest, such as the moon, Mars and asteroids, possess a sufficient atmosphere to sustain the consistently "high" surface temperatures found on Earth. Therefore, they can experience permanent cryogenic temperatures or dramatic cyclical changes in surface temperature. Characterization of physical properties (e.g., specific heat, thermal and electrical conductivity) over the entire temperature profile is important when planning a mission to a planetary surface; however, the impact on mechanical properties due to the introduction of icy deposits must also be explored in order to devise effective and robust excavation technologies. The Granular Mechanics and Regolith Operations Laboratory and the Cryogenics Test Laboratory at NASA Kennedy Space Center are developing technologies and experimental methods to address these challenges and to aid in the characterization of the physical and mechanical properties of regolith at cryogenic temperatures. This paper will review the current state of knowledge concerning planetary regolith at low temperature, including that of icy regolith, and describe efforts to manipulate icy regolith through novel penetration and excavation techniques.

\section{INTRODUCTION}

Water ice and other solid volatiles are known to exist on Mars and on various moons and asteroids throughout the solar system. Water is also believed to be frozen in the lunar regolith of permanently shadowed craters near the poles of the Moon. This particular finding has the potential to be game-changing because of the importance of accessing such a nearby resource located outside the Earth's gravity well. It will be important to ground-truth such planetary findings by sending spacecraft to robotically probe the regolith with instruments able to directly measure the ice content. However, 
this type of surface operation will be difficult to accomplish using a rover that does not have adequate weight to provide the necessary downforce in a low gravity environment.

During the Apollo program, it is known that the astronauts found it extremely difficult to obtain core samples in $1 / 6-\mathrm{G}$ gravity in the dense, frictional lunar regolith. The results of terrestrial experiments using JSC-1A lunar regolith simulant have shown the importance of testing lunar regolith containing even low concentrations of water ice, and the necessity of designing and testing flexible yet robust excavators (Gertsch 2006). Those experiments found that regolith with 0.6 to $1.5 \%$ ice behaves like weak shale or mudstone and is readily excavated; that regolith with $\sim 8.4 \%$ ice acts like moderate-strength limestones, sandstones, and shales that can be excavated using mechanical excavators; but the highest strength mix ( 10.6\% ice) behaves like strong limestone or sandstone and requires massive excavators. Similar work by Orbitec, Inc. found that water mixed with and compacted in JSC-1A lunar simulant at $8-10 \%$ (by volume) produced the hardest possible frozen samples for excavation. It will be even more difficult to take core samples, anchor into, and mine asteroids and small moons, like Phobos, or mine the hard, icy soil that may be found on the Moon.

In order to design and evaluate low-force penetrator and excavator systems for exploration on planetary surfaces, it is important to understand the geotechnical properties of planetary regolith (Alshibli 2009; Carrier 1991; Gamsky 2010; Klosky 2000; Zeng 2010), and to test these systems using terrestrial analogs of planetary icy regolith produced under relevant conditions. NASA Kennedy Space Center has developed techniques in recent years to excavate dry regolith simulant in large testbeds under ambient conditions, e.g. the Regolith Advanced Surface Systems Operations Robot [RASSOR] shown in Figure 1 (Mueller 2013).

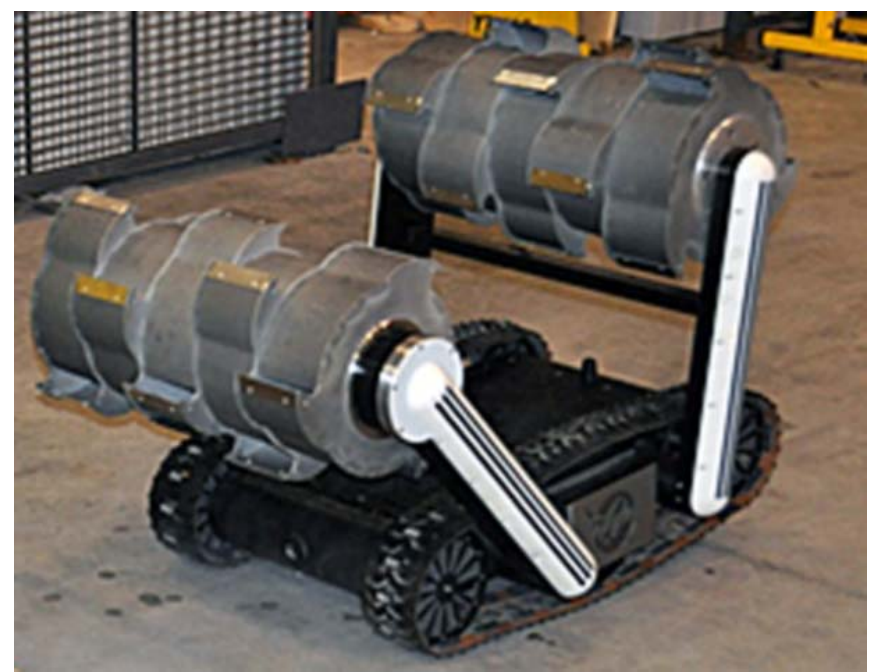

Figure 1. The NASA KSC robotic excavator known as RASSOR.

This paper will discuss the state of knowledge involving the formation of icy regolith in a relevant atmosphere, and describe ongoing efforts at KSC to produce large amounts of icy regolith in a vacuum environment that can be used to test 
prototype implements to excavate icy regolith on planetary bodies (e.g. Moon, Mars and asteroids), and measure its thermal properties and characterize its soil mechanics.

\section{STATE OF KNOWLEDGE}

Previous work conducted by Honeybee Robotics Spacecraft Mechanisms Corporation has demonstrated significant power savings can be achieved using a percussive cone penetrometer on dry lunar regolith simulant, which required only a small fraction of the force of an ordinary penetrometer (Zacny 2008). Honeybee Robotics and NASA Kennedy Space Center have also demonstrated that percussive penetration is a suitable method to insert instruments into a mixture of regolith and water ice in which the concentration of water varies (Metzger 2011). The published report of the latter experiments details how the measured penetration resistance varies with depth when a percussive cone penetrometer was applied solely under its own weight to a 1-meter deep column of a frozen mixture of lunar regolith simulant JSC-1A and water consisting of layers stacked in such a way that the water ice content varied from $0 \%$ in the top layer to $10 \%$ water by weight in the bottom layer. It should be noted that, on the Moon, the depth beneath the desiccated upper layers of lunar soil to reach the icy regolith in the polar regions is expected to be on the order of one-meter (Sanders 2012). From these previous geotechnical studies, it appears likely that even within dense layers of icy regolith, a system that combines percussive penetration with a companion sensor probe to directly detect the presence of water-ice has the potential to provide important ground truth data for exploration missions to planetary surfaces in search of water, whether the destination is the Moon, Mars, or asteroids.

In 2012, NASA Kennedy Space Center began using large amounts of icy regolith to support the development and testing of excavation tools for future planetary surface missions. The effectiveness of the digging implements needed to be measured using regolith containing water ice. The regolith chosen was a mineral aggregate material called BP-1, which KSC researchers had found in 2010 near the Black Point basalt flow in the San Francisco Volcanic Field in northern Arizona (Rahmatian 2010, Stoeser 2010). It was discovered in laboratory testing at KSC that the geotechnical properties of BP-1 are very similar to the higher fidelity lunar simulants, such as NU-LHT-2M and CHENOBI, apparently due to BP-1's lunar-like particle size distribution and its presumably jagged particle shapes, thus making it useful as a physical lunar regolith analog simulant. When dry BP-1 is mixed in a 30:1 ratio with water under ambient conditions, it was found to remain granular even after freezing by submerging it in liquid nitrogen (LN2) for ten minutes or more. It was then found that a hard block of icy regolith forms when a given amount of BP-1 was mixed in a 10:1 ratio with water (approximately 9\% water by volume), compacted into round stainless-steel duct end caps $7.5 \mathrm{~cm}$ tall by $20 \mathrm{~cm}$ diameter, and then frozen in liquid nitrogen (LN2) to temperatures as low as $83 \mathrm{~K}$ as measured using an embedded thermocouple located at the center of a block of icy regolith. Icy regolith with such a level of water ice content was selected by KSC to be an extreme test case for studying the effectiveness of implements intended to excavate hard icy regolith, albeit under ambient conditions. 
Batch production of six 21-liter trays of icy regolith was possible using a 50liter cement mixer to combine large quantities of BP-1 with water. A round foam plug was used over the mixer opening to contain the water during mixing. The moist BP-1 was hand scooped into stainless trays and hand compacted after filling. The filled trays with lids partly in place were separately wrapped in plastic to retain their moisture content, and then transported to the KSC Vibe Lab for vibratory compaction using a vertical shaker unit. An extra 20 pound metal weight was placed on top of the tray's lid to assist compaction. Batch production freezing down to $83 \mathrm{~K}$ of 6 trays of the BP-1 plus water mixture was then performed. One of the primary safety concerns in the process proved to be lifting the heavy frozen blocks out of a full LN2 cryo bath after freezing which was accomplished safely using a forklift. To separate a block of the icy regolith from its tray, a hand held heat gun was moved evenly over the bottom and sides of an overturned tray until the block of BP-1 dropped out as one piece.

Field testing of the RASSOR excavator was conducted both indoors and in an outdoor pit that contained the blocks of icy regolith made with BP-1. The RASSOR was operated in a double bucket dig mode and gravity off-loaded to simulate lunar gravity with its tracks in contact with frozen dirt. This preliminary testing served as a proof-of-concept that the rotating bucket drums of RASSOR could be used to break up the icy regolith for excavation regardless of the hardness.
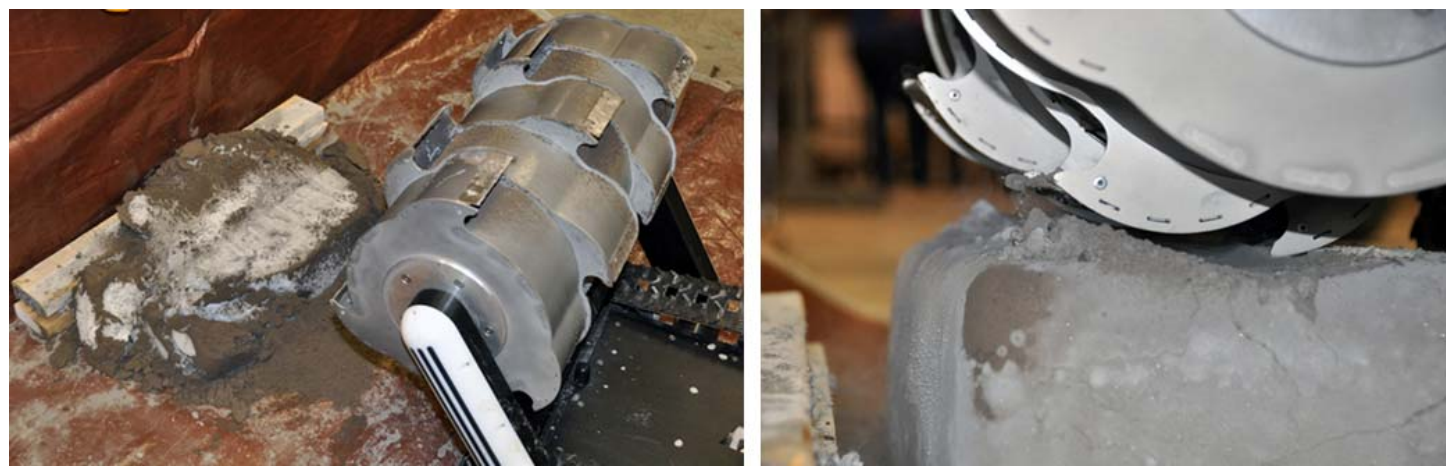

Figure 2. RASSOR's bucket drum is shown excavating icy regolith formed by cryo-freezing BP-1 simulant mixed with water using a 10:1 ratio of regolith to water.

\section{CURRENT ACTIVITIES INVOLVING ICY REGOLITH AT NASA KSC}

Some of the key objectives in exploring asteroids and the polar regions of the Moon and Mars include excavating icy regolith and then identifying and capturing the solid volatiles (e.g., water ice and carbon dioxide ice). Current activities at NASA KSC are aimed at demonstrating excavation techniques for collecting icy regolith containing water ice under relevant environmental conditions using suitable vacuum chamber systems. KSC is developing methods of producing large amounts of ice and regolith mixtures under low atmospheric pressure in large vacuum chambers with the capability of testing technologies for excavating regolith under simulated planetary surface environmental conditions. This will also allow excavation models to be tested for validity in experiments conducted under relevant atmospheric conditions so 
that the most efficient and effective excavation tools can be developed for surface operations including mining and sample capture on planetary bodies.

If the subsurface of a planetary body is divided into a warmer upper layer of dry porous regolith on top of a lower cold layer of regolith containing pores that can be filled with ice, then the thermal inertia in the lower layer can be many times greater than the upper layer due to the higher thermal conductivity of icy regolith (Rapp 2006). As gaseous volatiles in the atmosphere enter the pores of the granular top surface of regolith, gas molecules will diffuse into the regolith until they reach the ice table at the temperature and pressure at which solid ice begins to form. The ice table presents a barrier to continued downward diffusion of gaseous volatiles. On Mars, for example, atmospheric carbon dioxide gas and water vapor are each able to diffuse into the Martian regolith but the thermodynamic conditions on Mars are such that it is possible for water to solidify and form an ice table in subsurface regolith while carbon dioxide remains a gas except at the poles where conditions may support the formation of solid carbon dioxide as well.

Current KSC efforts are intended to demonstrate that a large amount of icy regolith can be formed under Martian atmospheric pressures. The work at KSC expands upon prior studies at Caltech (Hudson 2009, Siegler 2012) in which the formation of icy regolith was investigated using soda lime glass beads as a regolith simulant in a volume that contained a mass of $\sim 0.55 \mathrm{~kg}$.

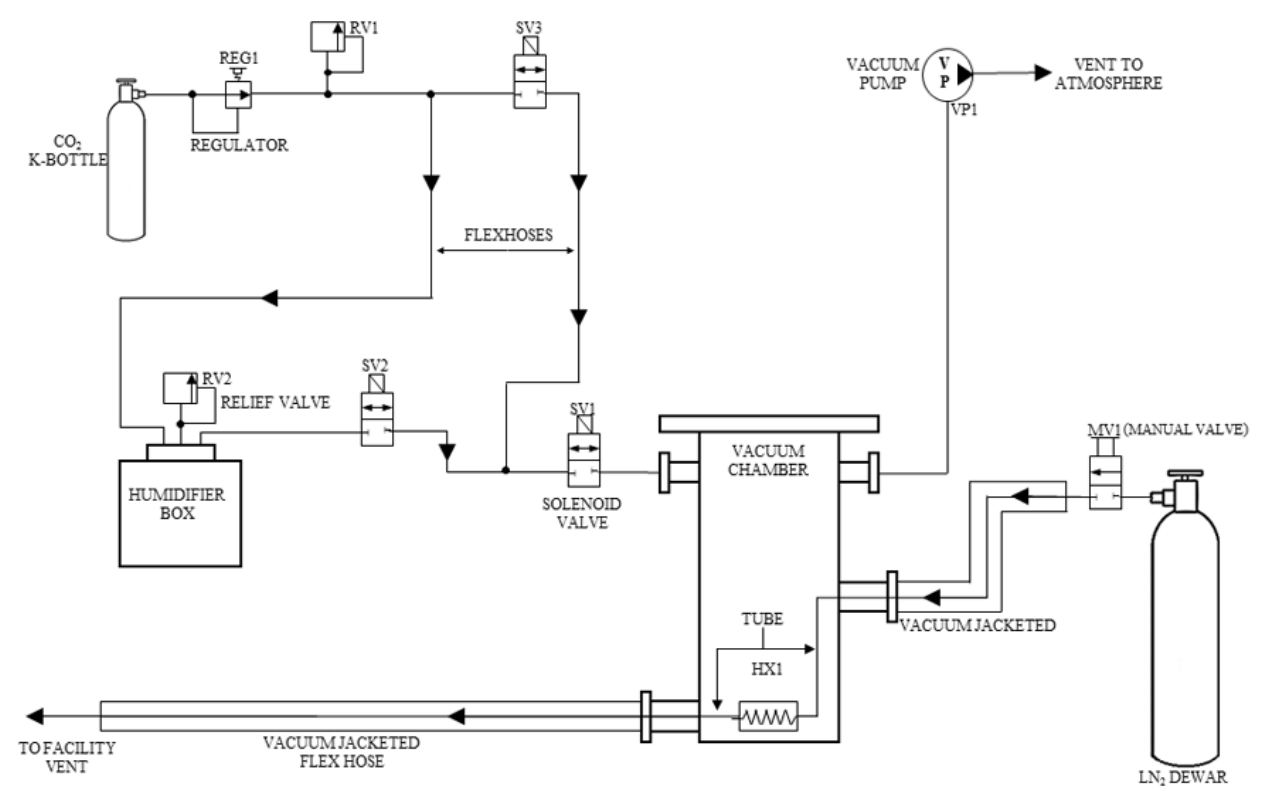

Figure 3. Schematic of Icy Regolith test setup at NASA KSC.

A schematic of the KSC setup is shown in Figure 3. The cryostat, shown in Figures 3 and 4, is being used at KSC for initial testing as a proof of concept demonstration. The cryostat encloses a cylindrical regolith sample holder the bottom of which is placed in thermal contact with a heat exchanger containing vacuumjacketed LN2 cryogenic cooling line which allows the regolith at the bottom of the 
sample holder to be held at a constant cold temperature while a resistive heater system keeps the top layer of regolith in the sample holder at a constant high temperature. Eventually, thermal equilibrium is reached in the dry simulant. But when water vapor is introduced into the system, the water molecules will diffuse from the warmer surface into the regolith until it encounters regolith that is cold enough for the water to freeze and fill the pores of the regolith. Once the ice table begins to form, no further diffusion into lower layers will occur. At this point, ice continues to grow upwards as the thermal conductivity of the icy regolith mixture increases. The regolith sample holder is 8 inches in diameter and 12 inches in height and is able to hold $\sim 15 \mathrm{~kg}$ of loosely compacted JSC-1A regolith simulant or other granular materials.
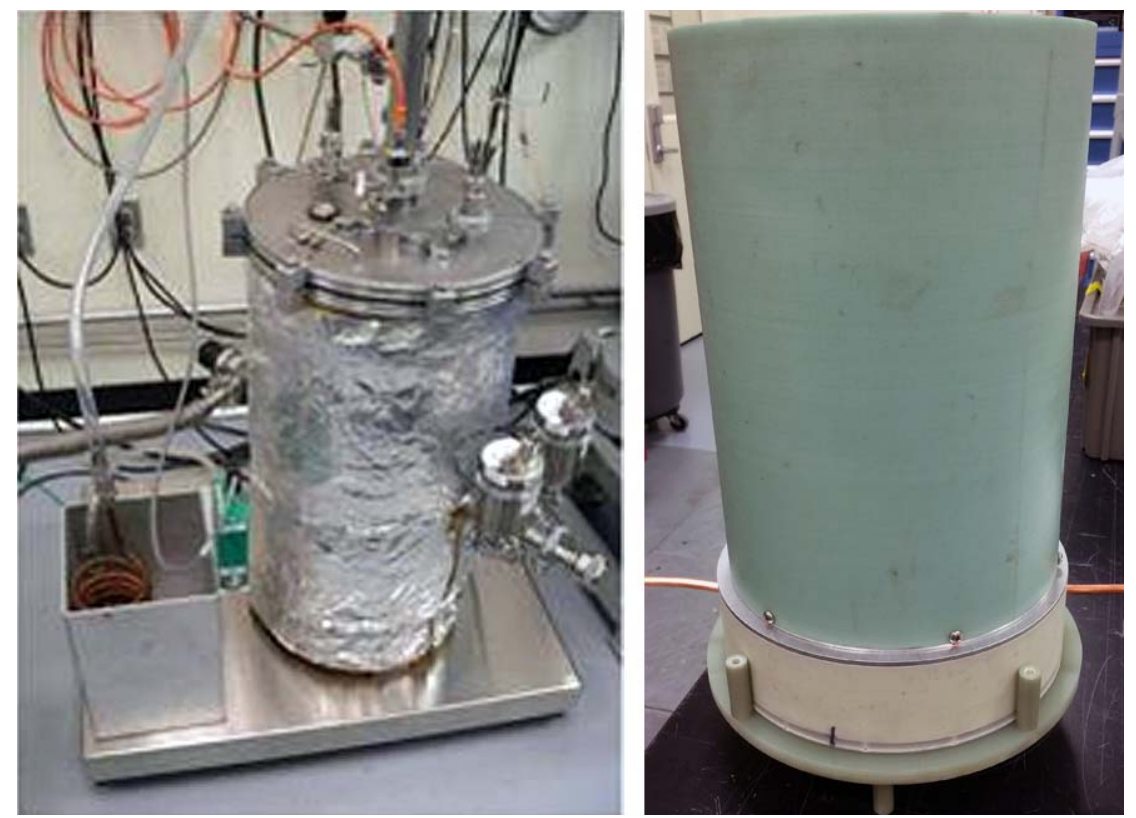

Figure 4. Cryostat (left) and Regolith Sample Holder (right) on Heat Exchanger.

KSC is now planning to expand this proof-of-concept setup using a cryostat into a large-scale regolith simulant testbed inside a chamber that holds $~ 1.3$ metric tons of planetary regolith simulant. This capability will allow for full-scale system testing of excavation and surface penetration techniques designed for planetary surface exploration that will allow icy regolith to form under relevant vacuum conditions for the Moon, Mars and asteroids.

\section{CONCLUSION}

NASA Kennedy Space Center is currently conducting studies in granular mechanics and surface regolith operations to test excavation systems and demonstrate the surface penetration of instruments into a large volume of icy regolith that is formed under low pressure conditions inside a vacuum chamber. Penetration testing is demonstrated using a cryostat chamber that holds $\sim 15 \mathrm{~kg}$ of icy regolith simulant formed under vacuum conditions by mixing water vapor with cryo-cooled volumes of regolith, such as JSC-1A, BP-1 and soda lime glass beads. Long-term goals at KSC 
call for testing full-scale excavation and surface penetration systems designed for planetary surface exploration using a large-scale regolith simulant testbed that can contains roughly 1.3 metric tons of regolith inside a chamber that will allow icy regolith to form under relevant vacuum conditions for studies involving the Moon, Mars and asteroids. This work will increase our scientific understanding of how icy regolith forms over time within the subsurface layers, and how the temperature and pressure conditions influence its thickness.

\section{ACKNOWLEDGMENTS}

We gratefully acknowledge support for this work from the Center Innovation Fund (CIF) under Karen Thompson, Center Chief Technologist at NASA Kennedy Space Center, and the support of Nancy Zeitlin, Technology Integration Manager at NASA KSC.

\section{REFERENCES}

Alshibli, Khalid A., and Alsidqi Hasan (2009), "Strength Properties of JSC-1A Lunar Regolith Simulant,” J. Geotech. and Geoenvir. Engineering 135 (5), 673-679.

Carrier, W. David, III, Gary R. Olhoeft and Wendell Mendell, "Physical Properties of the Lunar Surface," in Lunar Sourcebook, A User's Guide to the Moon, G. H. Heiken, D.T. Vaniman and B.M. French, eds., (Cambridge University Press, Melbourne, Australia, 1991), pp. 475 - 594.

Gamsky, Jacob N., and Philip T. Metzger (2010), "The Physical State of Lunar Soil in the Permanently Shadowed Craters of the Moon,” paper presented at Earth and Space 2010, 12th Biennial ASCE Aerospace Division International Conference on Engineering, Construction and Operations in Challenging Environments, Honolulu, HI, Mar. 14-17, 2010.

Gertsch, Leslie, Robert Gustafson, Richard Gertsch (2006), "Effect of water ice content on excavatability of lunar regolith," paper presented at Space Technology and Applications International Forum - STAIF 2006, AIP Conference Proceedings, vol. 813 (AIP, Melville, NY), pp. 1093-1100.

Hudson, T.L., Aharonson, O., and Schorghofer, N. (2009). "Laboratory experiments and models of diffusive emplacement of ground ice on Mars," J. of Geophys. Res. 114 (E1) CiteID E01002.

Klosky, J.L., S. Sture, H.-Y. Ko, and F. Barnes (2000), "Geotechnical behavior of JSC-1 lunar soil simulant,” J. Aerospace Eng. 13 (4), 133-138.

Metzger, P.T., Galloway, G.M., Mantovani, J.G., Zacny, K., and Craft, J. (2011). "Low Force Icy Regolith Penetration Technology.” NASA Technical Memorandum NASA/TM-2011-216302. 
Mueller, Robert P.; Cox, Rachel E.; Ebert, Tom; Smith, Jonathan D.; Schuler, Jason M.; Nick, Andrew J., "Regolith Advanced Surface Systems Operations Robot (RASSOR)," Aerospace Conference, 2013 IEEE , vol., no., pp.1,12, 2-9 March 2013. doi: 10.1109/AERO.2013.6497341

Rahmatian, L.A. and Metzger, P.T. (2010). "Soil Test Apparatus for Lunar Surfaces," 4th NASA/ARO/ASCE Workshop on Granular Materials in Lunar and Martian Exploration, pp. 239-253, (doi: http://dx.doi.org/10.1061/41096(366)25).

Rapp, Donald (2006). “Accessible Water on Mars,” JPL Report D-31343-Rev.7.

Sanders, G.B., Baird R.S., Rogers K. N., Larson W. E., Quinn J.W., Smith J.E., Colaprete A., Elphic R.C., and Picard M. (2012). "RESOLVE Lunar Ice/Volatile Payload Development and Field Test Status,” Presentation \#3046, Annual Meeting of the Lunar Exploration Analysis Group, Greenbelt, Maryland, 22-24 October 2012.

Siegler, Matthew; Aharonson, Oded; Carey, Elizabeth; Choukroun, Mathieu; Hudson, Troy; Schorghofer, Norbert; Xu, Steven (2012). "Measurements of thermal properties of icy Mars regolith analogs,” J. of Geophys. Res., 117(E3) CiteID E03001.

Stoeser, D.B., Wilson, S., and Rickman, D.L. (2010). "Preliminary geological findings on the BP-1 simulant." NASA Technical Memorandum NASA/TM-2010216444.

Zacny K., Fong T., Wilson J., Lee S., Kobayashi L., Deans M., Ashley A., and Santoro C. (2008). "Percussive Dynamic Cone Penetrometer for Geotechnical Surface Assessment with a Planetary Rover.” NASA Lunar Science Institute Conf., LPI Contribution No. 1415, abstract no. 2138.

Zeng, Xiangwu, Chunmei He, Heather Oravec, Allen Wilkinson, Juan Agui, and Vivake Asnani (2010), “Geotechnical Properties of JSC-1A Lunar Soil Simulant,” J. Aerosp. Engineering 23 (2), 111-116. 